\title{
SYNTHESIS OF SPHERICAL SILICA PARTICLES WITH 3-MERCAPTOPROPYL GROUPS IN THEIR SURFACE LAYER
}

\author{
Nazarchuk G. I., Melnyk I. V., Zub Yu. L.* \\ Chuiko Institute of Surface Chemistry, National Academy of Science of Ukraine, \\ 17, General Naumov Street, Kyiv 03164 Ukraine; \\ *E-mail: zub_yuriy@isc.gov.ua, phone+38(044)4229630,fax: +38(044)4243567
}

\begin{abstract}
Spherical silica particles were synthesized by modified Stöber method using tetraethoxysilane and 3mercaptopropyltrimethoxysilane (MPTMS). It was shown that their size $(500-760 \mathrm{~nm})$ depends on the nature of the catalyst used in the prehydrolysis of MPTMS (at constant alkoxysilanes ratio). Elemental analysis, thermogravimetry and IR spectroscopy data indicate the presence of thiol groups $(2.8 \mathrm{mmol} / \mathrm{g})$ in the surface layer. It was established that obtained nanospherical silica particles can adsorb $\operatorname{Ag}(\mathrm{I})$ ions from their acidified aqueous solutions.
\end{abstract}

Keywords: silica spheres, modified Stöber method, 3-mercaptopropyl groups.

\section{Introduction}

Silica has been widely used in industry [1]. However, the increasing attention is recently drawn to micro-and nanoparticles of silicon oxide, primarily of spherical shape, which are used in many areas, such as ceramics production, chromatography, chemical sensory, biomedicine [2,3]. In addition, they increasingly began to be used as stabilizers, emulsifiers, binders, in creating coatings for glass, etc. [4]. Necessity to develop new ways to obtain these materials and more detailed study of their structure and properties has increased with the increasing demand on the silica particles for high-tech industries (such as pharmaceutics and biotech). Nano- and microparticles of silica with functional groups in the surface layer are of special interest. Functional groups endow the particles with new specific properties, which significantly expands the practical application of such materials [5-7].

Monodisperse silica spheres 50-1700 $\mathrm{nm}$ in size are usually obtained by Stöber method, i.e. hydrolytic polycondensation of tetraethoxysilane in the environment of low-molecular alcohol (catalyst - ammonium hydroxide) [8]. The same method in one- and two-stage version was used for the synthesis of silica spheres containing functional groups in the surface layer [9-11].

The aim of this work is to develop one-stage method of obtaining monodisperse silica spheres with complexing groups in the surface layer. The synthesis of silica particles with 3-mercaptopropyl groups $\equiv \mathrm{Si}\left(\mathrm{CH}_{2}\right)_{3} \mathrm{SH}$ is considered as the first example.

\section{Experimental}

The following reagents were used for the synthesis of spherical silica particles: tetraethoxysilane (TEOS), $\mathrm{Si}\left(\mathrm{OC}_{2} \mathrm{H}_{5}\right)_{4}\left(99 \%\right.$, Aldrich); 3-mercaptopropyltrimethoxysilane (TMPTMS), $\left(\mathrm{CH}_{3} \mathrm{O}\right)_{3} \mathrm{Si}\left(\mathrm{CH}_{2}\right)_{3} \mathrm{SH}(95 \%$, Aldrich); ammonium hydroxide (25\%, Khimlaborreaktyv, Ukraine); 0.1 M hydrochloric acid (Khimlaborreaktyv, Ukraine); $\mathrm{NH}_{4} \mathrm{~F}$ (98\%, Fluka); absolutized ethanol.

Synthesis of silica spheres with 3-mercaptopropyl groups (TEOS/MPTMS $=3: 1$ (mol.)). Sample 1. $21 \mathrm{~cm}^{3}$ of $25 \% \mathrm{NH}_{4} \mathrm{OH}$ was dissolved in $40 \mathrm{~cm}^{3}$ of ethanol with stirring, after that $4.5 \mathrm{~cm}^{3}(0.3 \mathrm{~mol})$ of TEOS was added. Opalescence appeared in $2 \mathrm{~min}$. Suspension was stirred for 1 hour. Then $1.2 \mathrm{~cm}^{3}(0.1 \mathrm{~mol})$ of MPTMS was added and stirring continued for $24 \mathrm{hs}$. In this case, there was further increase in opalescence. The resulting precipitate was centrifuged at $7000 \mathrm{rpm}$ for $15 \mathrm{~min}$. The filtrate was elutriated and a new portion of ethanol was added $\left(25 \mathrm{~cm}^{3}\right)$, treated with ultrasound for $3 \mathrm{~min}$. and again centrifuged. The procedure was repeated two more times. The obtained white precipitate was dried in vacuum: $1 \mathrm{~h}-$ at room temperature, $1 \mathrm{~h}-$ at $50^{\circ} \mathrm{C}, 4 \mathrm{hs}-$ at $100^{\circ} \mathrm{C}$. The yield was $1.86 \mathrm{~g}$.

Considering sample 2, first $1.2 \mathrm{~cm}^{3}(0.1 \mathrm{~mol})$ of MPTMS was dissolved in $4 \mathrm{~cm}^{3}$ of ethanol and then $2.5 \mathrm{~cm}^{3}$ of $0.1 \mathrm{M} \mathrm{HCl}$ was added. After stirring for $1 \mathrm{~h}$, the resulting solution was added to another solution $-4.5 \mathrm{~cm}^{3}(0.3 \mathrm{~mol})$ of TEOS in $40 \mathrm{~cm}^{3}$ of ethanol and $21 \mathrm{~cm}^{3}$ of $25 \% \mathrm{NH}_{4} \mathrm{OH}$, which previously also stirred for $1 \mathrm{~h}$. Suspension was mixed 23 $\mathrm{h}$ more and further processed as described for sample 1 . The yield was $2.13 \mathrm{~g}$.

In case of sample 3, first $1.2 \mathrm{~cm}^{3}(0.1 \mathrm{~mol})$ of MPTMS was dissolved in $4 \mathrm{~cm}^{3}$ of ethanol and the solution of $\mathrm{NH}_{4} \mathrm{~F}\left(5 \mu \mathrm{g} \mathrm{NH} \mathrm{F}_{4} \mathrm{~F}\right.$ in $2.5 \mathrm{~cm}^{3} \mathrm{H}_{2} \mathrm{O}$ ) was added. The obtained (within $2 \mathrm{~min}$ ) clear solution was added to TEOS (which was also previously stirred for $1 \mathrm{~h}$ in alcohol with ammonia) and suspension was mixed for $23 \mathrm{~h}$ more. Further processing was carried as described for sample $\mathbf{1}$. In this case, the formed precipitate featured curd-like consistency. The yield was $2.06 \mathrm{~g}$. 
Elemental analysis was performed in the certified analytical laboratory of the Institute of Organic Chemistry of NAS of Ukraine. IR spectra were recorded on Thermo Nicolet Nexus Fourier-transform infrared spectrometer in the 400-4000 cm $\mathrm{cm}^{-1}$ range, working in "Nexus Smart Collector" mode and averaging 50 scans with a resolution of $8 \mathrm{~cm}^{-1}$. The samples were previously ground with solid KBr ("Fluka", for IR spectroscopy) at a ratio of 1:30. The spectra of the initial alkoxysilanes were recorded in liquid form between the KRS plates.

The scanning-electron microphotographs were taken with Scanning Electron Microscope JSM 6060 LA (Jeol, Japan) using secondary electrons at an accelerated voltage of $30 \mathrm{kV}$. Samples were stuck on the objective table. Their surface was coated in vacuum with thin continuous layer of gold by cathodic sputtering to prevent the accumulation of surface charges and to receive a contrasting picture.

Thermogravimetric (TG) and differential thermal analyses (DTA) were performed on a Paulik-Paulik-Erde (Q-1500D) instrument operating in the $20-1000^{\circ} \mathrm{C}$ range, with a heating rate of $10^{\circ} \mathrm{C} / \mathrm{min}$. The sensitivity of thermal scales was $\pm 0.1 \mathrm{mg}$.

The measurement of low-temperature $\left(-196^{\circ} \mathrm{C}\right)$ nitrogen adsorption and desorption isotherms was carried out on “Kelvin-1042” (Costech Microanalytical) analyzer. Sample outgassing was achieved at $100^{\circ} \mathrm{C}$ during 2 hs. Data were processed using BET equation [12].

Investigation of $\mathrm{Ag}^{+}$sorption from nitrate solutions was carried out in static mode $(\mathrm{m}=0.05 \mathrm{~g}( \pm 0.0005), \mathrm{V}=$ $20 \mathrm{~cm}^{3}$, initial solution $\mathrm{pH} \sim 2, \mathrm{t}=20^{\circ} \mathrm{C}$ ). The concentration of $\mathrm{Ag}^{+}$in initial solution and filtrate were determined by atomic absorption method at the spectrophotometer C-115-M1.

\section{Results and discussion}

Stöber et al. in [8] showed that 2 hs mixing time is sufficient for obtaining spherical silica particles by hydrolytic polycondensation of TEOS in ammonium alkali medium. Moreover, they found that sometimes particles reach their final size during the first $15 \mathrm{~min}$. In [13] during the synthesis of silica spheres with 3-aminopropyl groups in the surface layer, the suspension was stirred for 1 hour. For this reason, at first, this term was used for suspension stirring after MPTMS addition (synthesis of sample 1). Indeed, in this case the formation of spherical particles is observed (Fig. 1a), but their IR spectrum lacks the absorption band of valence $v(\mathrm{SH})$ vibrations. It means, that thiol groups are practically absent in the surface layer of the particles. That is why suspension stirring time was increased to 24 hs for further syntheses. In addition, we established that using two-component systems (TEOS/MPTMS), the best results are obtained during successive addition of reacting components with prehydrolysis of trifunctional silane.

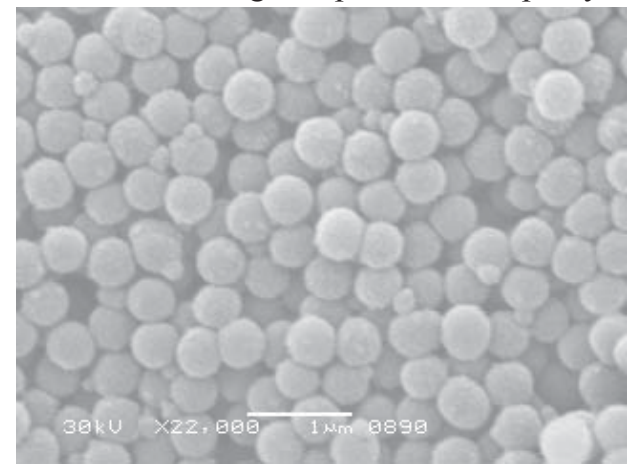

$a$

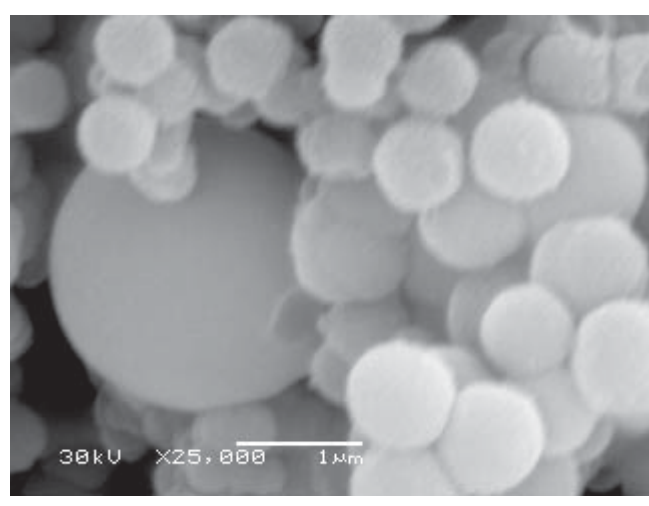

c

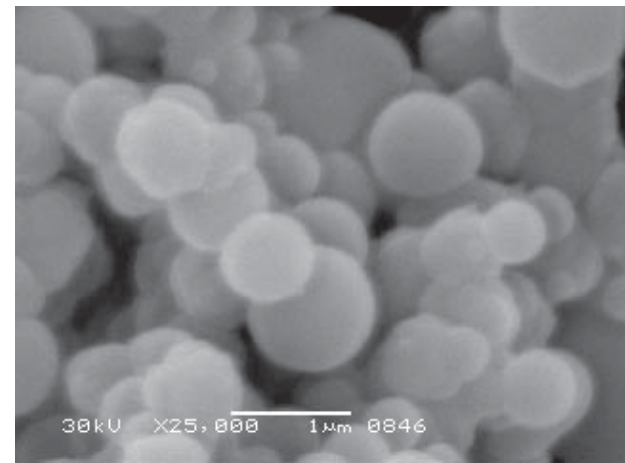

$b$

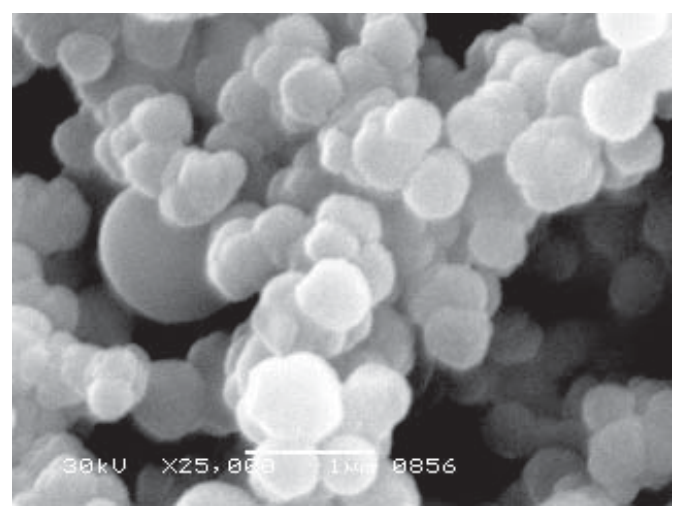

$d$

Figure 1. SEM microphotographs for: $a$-sample without thiol groups, $b$ - sample 1, $c$ - sample $\mathbf{2}, d$ - sample $\mathbf{3}$. 
Fig. 1 represents SEM microphotographs of synthesized samples. Note that Fig. 1a shows silica spheres without 3-mercaptopropyl groups. Their size is about $410 \mathrm{~nm}$. Silica spheres with thiol groups always have greater diameter. So, according to Fig. 1b, the average diameter of silica spheres in sample $\mathbf{1}$ is $740 \mathrm{~nm}$. In the case of samples 2 and $\mathbf{3}$ (Fig. 1c and 1d, respectively), there are some isolated spheres that differ in size significantly. If they are taken out of consideration, the average size of silica spheres is $760 \mathrm{~nm}$ for sample 2, and $510 \mathrm{~nm}$ for sample 3. Thus, varying the nature of catalyst used in the hydrolysis of MPTMS, one can obtain spherical silica particles with sizes in the range $500-760 \mathrm{~nm}$.

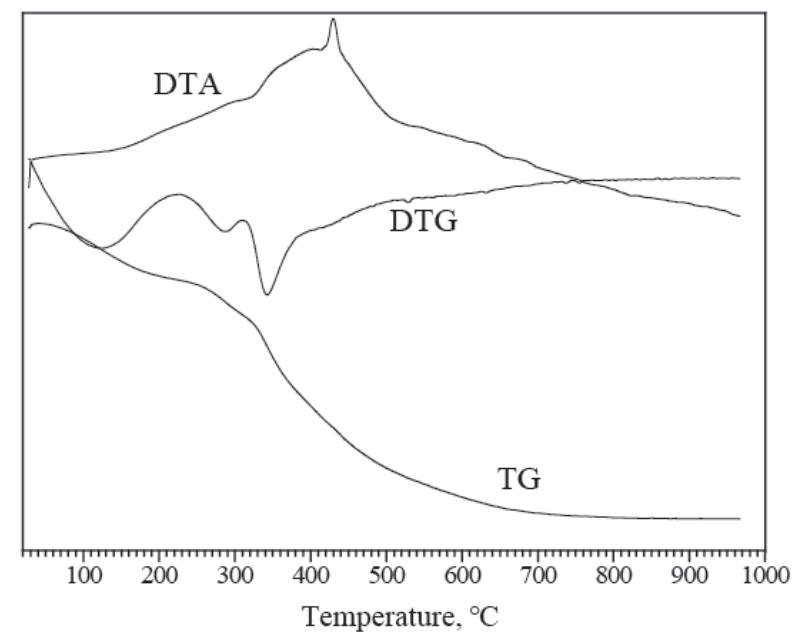

Figure 2. Thermoanalytical curves for sample 3.

Estimation of specific surface area has shown that it is in the range of $7-9 \mathrm{~m}^{2} / \mathrm{g}$ for synthesized hybrid materials.

The data of element analysis testified he presence of thiol groups in the obtained powdery materials. Thus, sulfur content is $9.06 \%$ for sample $\mathbf{2}$. On this basis we can calculate the content of functional groups (2.8 $\mathrm{mmol} / \mathrm{g})$. It is close to the content, calculated from the molar ratio of reacting alkoxysilanes $(3.6 \mathrm{mmol} / \mathrm{g})$.

Thermogravimetry data indicate the presence of carbofunctional groups in the synthesized samples. Thus, the DTG curve of sample 3 (Fig. 2) has three exoeffects. The first centered around $120^{\circ} \mathrm{C}$ is associated with removal of adsorbed residual water (weight loss is $\sim 5 \%$ ). Second exoeffect on the DTG curve is centered around $280^{\circ} \mathrm{C}$, which, likely, corresponds to the removing (or oxidation) of thiol-containing fragments $-\mathrm{H}_{2} \mathrm{~S}_{\text {and }} \mathrm{CH}_{3} \mathrm{SH}$ [14] (weight loss is $4 \%$ ). The third exoeffect with a maximum at $350^{\circ} \mathrm{C}$ corresponds to more significant mass loss $(19,1 \%)$, indicating the complete burnout of the organic residues. Therefore, endothermic effect is observed in the DTA curve in the range $350-450^{\circ} \mathrm{C}$ (Fig. 2). The total weight loss during the second and the third stages $(3.1 \mathrm{mmol} / \mathrm{g})$ agrees with the content of functional groups, calculated on the basis of elemental analysis (see above). TGA data (Fig. 2) make it easy to conclude that the destruction of the organic layer begins at $230^{\circ} \mathrm{C}$. This is also typical for xerogels with thiol groups [15].

The presence of 3-mercaptopropyl groups in the surface layer is also confirmed by IR spectroscopy data. Thus, in the FT-IR spectra of all three samples (Fig. 3) the absorption band of medium intensity at $2555 \mathrm{~cm}^{-1}$, attributable to $v(\mathrm{SH})$, is clearly recorded, and a low-intense absorption band at $\sim 685 \mathrm{~cm}^{-1}$ is associated with valence vibrations of C-SH [16]. There is also a group of absorption bands at 2800-3000 $\mathrm{cm}^{-1}$, which can be attributed to the valence vibration $v_{\text {s.as }}(\mathrm{CH})$ of the propyl chain. Its presence is also reflected in the presence of a group of bands of weak intensity in 1300-1490 $\mathrm{cm}^{-1}$ region (Fig. 3). In addition, the IR spectra of obtained materials have the most intense absorption band at 1000-1200 $\mathrm{cm}^{-1}$ with a high-frequency shoulder (Fig. 3). Its occurrence is associated with the existence of three-dimensional network of polysiloxane bonds, $\equiv \mathrm{Si}-\mathrm{O}-\mathrm{Si} \equiv[17]$. Since all the synthesized samples contain water, there is a characteristic absorption band of deformation vibrations of $\mathrm{H}_{2} \mathrm{O}$ molecules at $\sim 1630 \mathrm{~cm}^{-1}$, and a broad intense absorption band above $3000 \mathrm{~cm}^{-1}$ due to the $\mathrm{OH}^{-}$groups vibrations of water molecules participating in the formation of hydrogen bonds. There also should be mentioned the presence of characteristic absorption band of valence vibration for silanol groups at $\sim 3625 \mathrm{~cm}^{-1}$. Thus, the obtained spherical particles are composed of polysiloxane network with attached carbofunctional groups. Besides, there also can be an insignificant number of surface alkoxygroups, which is indicated

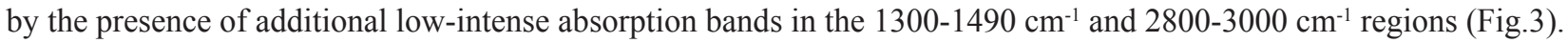




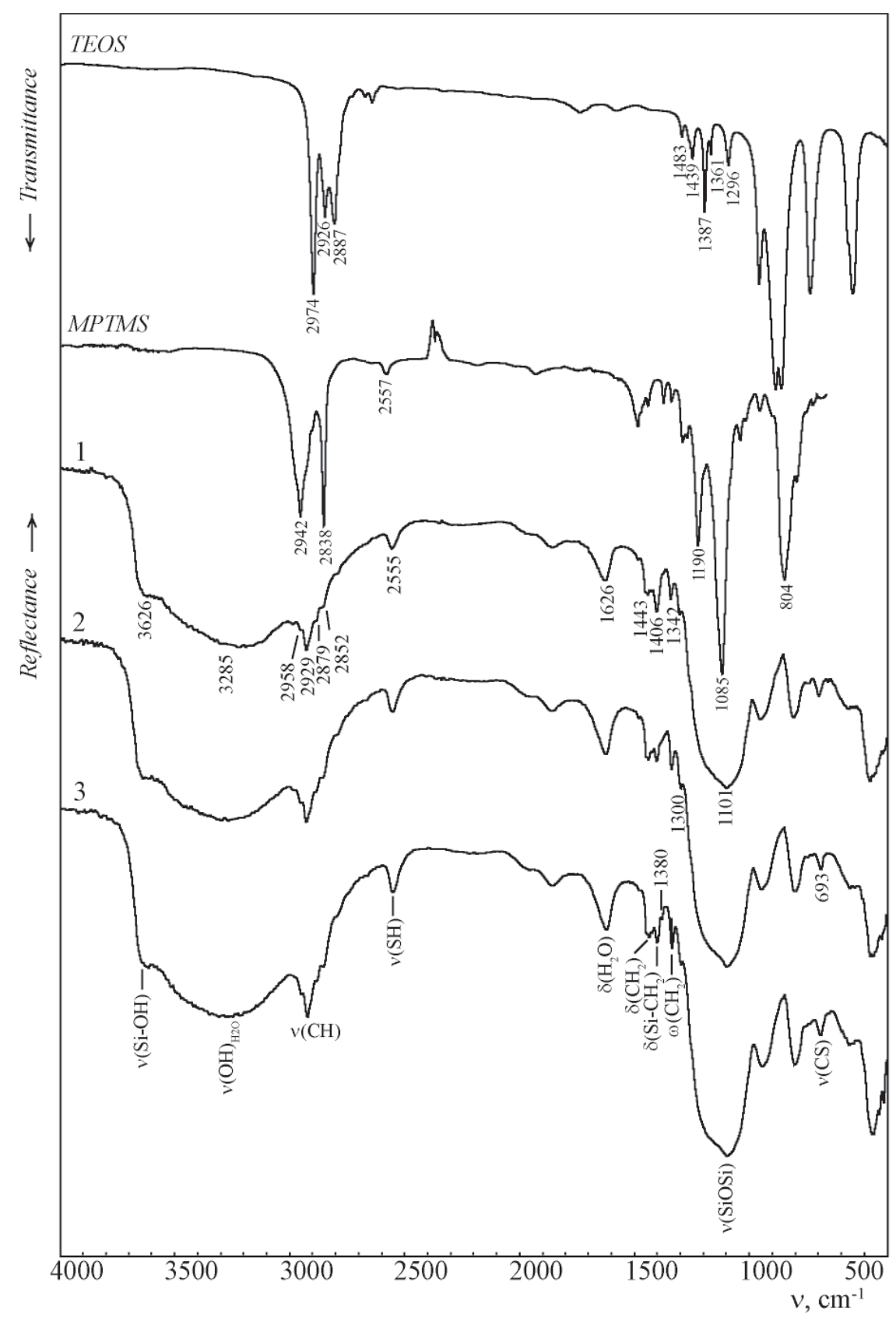

Figure 3. IR spectra of initial alkoxysilanes and synthesized spherical materials.

Sorption properties of functionalized spherical silica particles to silver(I) ions were investigated for sample 2. It was experimentally determined that $1.28 \mathrm{mmol} / \mathrm{g}$ of silver (I) ions can be adsorbed for $1 \mathrm{~h}$. It means that the obtained materials could be perspective in the sorption of heavy metal ions from aqueous solutions.

\section{Conclusions}

The procedure of synthesis of silica spheres with 3-mercaptopropyl groups in their surface layer using twocomponent system (TEOS and MPTMS) was developed. SEM microphotographs showed the formation of spherical particles with sizes in the range 500-760 nm, which is determined by the nature of the catalyst used in the reaction of MPTMS hydrolysis. IR spectra indicate the presence of thiol groups and three-dimensional network of polysiloxane bonds in samples. The obtained silica spheres with 3-mercaptopropyl surface layer can sorb Ag(I) ions from their acidified aqueous solutions.

\section{Acknowledgements}

We express our gratitude to the Ukrainian State Program "Nanotechnologies and Nanomaterials" (Project No. 6.22.5.42) for financial support of the present work. 


\section{References}

[1]. Ayler, R. The Chemistry of Silica. Wiley Interscience: New York, 1979.

[2]. Ma, Y.; Qi, L.; Ma, J.; Wu, Y.; Lin, O.; Cheng, H. Large-pore mesoporous silica spheres: synthesis and application in HPLC. Colloids Surf. A 2003, 229, pp.1-8.

[3]. Roque-Malherbe, R.; Marquez, F. Synthesis and characterization of silica sphere-packing mesoporous materials. Surface and Interface Analysis. 2005, 37, pp.393-397.

[4]. Zhang, Y.; Jin, Y.; Yu, H.; Dai, P.; Ke, Y.; Liang, X. Talanta. 2010, 81, pp.824-830.

[5]. Wu, Z.; Han, H.; Han, W.; Kim, B.; Ahn, K.H.; Lee, K. Controlling the hydrophobicity of submicrometer silica spheres via surface modification for nanocomposite applications. Langmuir, 2007, 23, pp.7799-7803.

[6]. Wu, Z.; Xiang, H.; Kim, T.; Chun, M.S.; Lee, K. Surface properties of submicrometer silica spheres modified with aminopropyltriethoxysilane and phenyltriethoxysilane. J. Colloid Interface Sci., 2006, 304, pp.119-124.

[7]. Marquez, M.; Grady, B.P.; Robb, I. Different methods for surface modification of hydrophilic particulates with polymers. Colloids Surf. A, 2005, 266, pp.18-31.

[8]. Stöber, W.; Fink, A.; Bohn, E. Controlled Growth of monodisperse silica spheres in the micron size range. J. Colloid Interface Sci. 1968, 26, pp.62-69.

[9]. Toepfer, O.; Schmidt-Naake, G. Surface - functionalized inorganic nanoparticles in miniemulsion polymerization. Macromol. Symp. 2007, 248, pp.239-248.

[10]. Deng, T.-S. et al. Journal of Colloid and Interface Science 2009, 329, pp.292-299.

[11]. Berriozabal, G.; Yolanda, R. de Miguel. Synthesis and characterization of silica nanoparticles bearing different functional groups obtained via a two-stage method. Phys. Status Solidi. 2010, pp.2692-2696

[12]. Brunauer, J.S.; Emmet, P.H.; Teller, E. J. Amer. Chem. Soc. 1938, 60, pp.309- 319.

[13]. The Colloid Chemistry of Silica (Ed. By H.E.Bergna) // Adv.in Chem.Series 234, Amer.Chem.Sci., Washington, DC 1994, pp.83-111.

[14]. Diaz, I.; Mohino, F.; Perez-Pariente, J.; Sastre, E. Thermochim. Acta. 2004, 413, pp.201-207.

[15]. Dobryanska, H. I.; Melnyk, I. V.; Zub, Yu. L.; Chuiko, A. A.; Barcrak, M.; Dabrowski, A. The influence of the $\mathrm{Si}\left(\mathrm{OC}_{2} \mathrm{H}_{5}\right)_{4} /\left(\mathrm{CH}_{3} \mathrm{O}\right)_{3} \mathrm{Si}\left(\mathrm{CH}_{2}\right)_{3} \mathrm{SH}$ ratio on the structure-adsorption characteristics of xerogels formed and accessibility of functional groups in their surface layers. J. Phys. Chem. 2006, 80 (6), pp. 939-944 (in Russ.).

[16]. Lin-Vien, D.; Colthup, N.B.; Fateley, W.G.; Grasselli, J.G. The Handbook of Infrared and Raman CharacteristicFrequencies of Organic Molecules. L.: Acad. Press, 1991.

[17]. Finn, L.P.; Slinyakova, I.B. The structure and thermal decomposition of polyorganosiloxane xerogels, as shown by IR spectroscopy. Colloid. J.. 1975, V. 37, № 4, pp.723-729 (in Russ.). 